Pacific Journal of Mathematics

CHEBYSHEV CENTERS AND UNIFORM CONVEXITY 


\section{CHEBYSHEV CENTERS AND UNIFORM CONVEXITY}

\section{DAN AMIR}

If $E$ is a uniformly convex Banach space and $T$ is any topological space, then in the space $X=C(T, E)$ of $E$-valued bounded continuous functions on $E$, every bounded set has a Chevyshev center. Moreover, the set function $A \rightarrow Z(A)$, corresponding to $A$ the set of its Chebyshev centers, is uniformly continuous on bounded subsets of the space $\mathscr{B}(X)$ of bounded subsets of $X$ with the Hausdorff metric. This is contrasted with the fact that a normed space $X$ in which $Z(A)$ is a singleton for every bounded $A$ is uniformly convex iff $A \rightarrow Z(A)$ is uniformly continuous on bounded subsets of $\mathscr{B}(X)$.

Let $(X, d)$ be a metric space. Denoto by $\mathscr{B}(X)$ the space of nonempty bounded subsets of $X$ and let $h$ be the Hausdorff semimetric on $\mathscr{B}(X)$ :

$$
h(A, B)=\max \left(\sup _{u \in A} \inf _{v \in B} d(u, v), \sup _{v \in B} \inf _{u \in A} d(u, v)\right) .
$$

For $x \in X, r \geqq 0$, let $B(x, r)=\{y \in X ; d(x, y) \leqq r\}$ be the closed $r$-ball around $x$. For $A \in \mathscr{B}(X)$ and $x \in X$ denote $r(x, A)=\inf \{r \geqq 0$; $B(x, r) \supset A\}, r(A)=\inf _{x \in X} r(x, A)$ is the Chebyshev radius of $A$, and $Z(A)=\{x \in X ; r(x, A)=r(A)\}$ is the set of Chebyshev centers of $A$. For $Y \subset X$ we can consider also the relative Chebyshev radius of $A$ in $Y, r_{Y}(A)=\inf _{y \in Y} r(y, A)$, and the set of relative Chebyshev centers of $A$ in $Y, Z_{Y}(A)=\left\{y \in Y ; r(y, A)=r_{Y}(A)\right\}$. In the case that $A=\{x\}$ is a singleton, then $Z_{Y}(A)$ is just the set of best approximations in $Y$ to $x, P_{Y} x$.

We say that $X$ admits centers if every bounded set in $X$ has Chebyshev centers. The classical Banach spaces, i.e., the spaces $L_{p}(\mu), 1 \leqq p \leqq \infty$, over any measure space and the spaces $C(T)$ of continuous real-valued functions on compact Hausdorff $T$, admit centers ([1], [3]). However, Garkavi ([1]) gave an example of a 3point set in a maximal subspace $H$ of $C[0,1]$ which has no Chebyshev center in $H$. The problem of characterizing all Banach space which admit centers is still open.

Ward ([5]) proved that the space $C(T, E)$ of $E$-valued bounded continuous functions on the topological space $T$, with the norm $\|x\|=\sup _{t \in T}\|x(t)\|$, admits centers in each of the following two cases: (a) $E$ is a finite-dimensional strictly convex (hence uniformly convex) normed space and $T$ is paracompact. (b) $E$ is a Hilbert 
space and $T$ is normal. Ward asked whether both results can be strengthened by taking in (b) any uniformly convex Banach space $E$. Our first result shows that the answer is in the affirmative.

We use the following characterization of uniform convexity, due to Ruston ([4]). We include a proof for completeness sake.

1. Lemma. A normed space $E$ is uniformly convex iff for every $\varepsilon>0$ there is $\delta^{\prime}(\varepsilon)>0$ such that if $x, y \in E$ and $\phi \in E^{*}$ are such that $\|x\|=\|y\|=1=\|\phi\|=\phi(y)$ and $\phi(x)>1-\delta^{\prime}(\varepsilon)$, then $\|x-y\|<\varepsilon$. We can take, of course, $\delta^{\prime}(\varepsilon) \leqq 1 / 2 \varepsilon$.

Proof. If $E$ is uniformly convex, then $\delta(\varepsilon) \equiv \inf \{1-\|(u-v) / 2\|$; $\|u\|=\|v\|=1,\|u-v\| \geqq \varepsilon\}$ is positive. Take $\delta^{\prime}(\varepsilon)<2 \delta(\varepsilon)$. If $\|x-y\| \geqq \varepsilon$ then $\|(x+y) / 2\| \leqq 1-\delta(\varepsilon)$ hence $(\phi(x)+1) / 2=\phi((x+y) / 2) \leqq$ $1-\delta(\varepsilon)$ and $\phi(x) \leqq 1-2 \delta(\varepsilon)<1-\delta^{\prime}(\varepsilon)$.

Conversely, we claim that $\delta(\varepsilon) \geqq \delta^{\prime}(\varepsilon / 4)$. Indeed, if $\|x\|=\|y\|=1$ and $\|x-y\|>\varepsilon$, take $\phi \in E^{*}$ with $\|\phi\|=1, \phi(x+y)=\|x+y\|$. Then either $\|(x+y) / 2\|<1-\varepsilon / 4 \leqq 1-\delta^{\prime}(\varepsilon / 4)$, or $\quad\|(x+y) / 2\| \geqq 1-\varepsilon / 4$, hence $\|(x+y) / 2-(x+y) /\| x+y\|\| \leqq \varepsilon / 4$ and $\|x-(x+y) /\| x+y\|\| \geqq$ $\varepsilon / 4,\|y-(x+y) /\| x+y\|\| \geqq \varepsilon / 4$, hence $\phi(x) \leqq 1-\delta^{\prime}(\varepsilon / 4), \quad \phi(y) \leqq 1-$ $\delta^{\prime}(\varepsilon / 4)$ and $\|x+y\|=\phi(x+y) \leqq 2\left(1-\delta^{\prime}(\varepsilon / 4)\right)$.

2. Theorem. If $E$ is a uniformly convex Banach space and $T$ is any topological space, then $C(T, E)$ admits centers.

Proof. Given any bounded $A \subset C(T, E)$ we may assume, without loss of generality, that $r(A)=1$. Given $\varepsilon>0$, choose any $f_{0} \in C(T, E)$ with $r\left(f_{0}, A\right) \leqq 1+\delta^{\prime}(\varepsilon)$. We claim that there is $f_{1} \in C(T, E)$ with $r\left(f_{1}, A\right) \leqq 1+\delta^{\prime}(\varepsilon / 2)$ and $\left\|f_{1}-f_{0}\right\| \leqq 2 \varepsilon$. Indeed, take any $g \in C(T, E)$ with $r(g, A) \leqq 1+\delta^{\prime}(\varepsilon / 2)$ and define:

$$
\begin{aligned}
& \beta(t)=\left\{\begin{array}{lll}
1 & \text { if } & \left\|g(t)-f_{0}(t)\right\| \leqq 2 \varepsilon \\
\frac{2 \varepsilon}{\left\|g(t)-f_{0}(t)\right\|} & \text { if } \quad\left\|g(t)-f_{0}(t)\right\|>2 \varepsilon
\end{array}\right. \\
& f_{1}(t)=f_{0}(t)+\beta(t)\left(g(t)-f_{0}(t)\right) .
\end{aligned}
$$

Clearly, $f_{1} \in C(T, E)$ and $\left\|f_{1}-f_{0}\right\| \leqq 2 \varepsilon$. Take any $a \in A$. We have to show that $\left\|f_{1}(t)-a(t)\right\| \leqq 1+\delta^{\prime}(\varepsilon / 2)$. This is clear if $\beta(t)=1$, since then $f_{1}(t)=g(t)$, or if $\beta(t)<1$ but $\|g(t)-a(t)\| \geqq\left\|f_{0}(t)-a(t)\right\|$, since $f_{1}(t)$ lies on the segment $\left[f_{0}(t), g(t)\right]$. Therefore we may assume $1+\delta^{\prime}(\varepsilon) \geqq\left\|f_{0}(t)-a(t)\right\|>\|g(t)-a(t)\|$. Denote $u=f_{0}(t)-a(t), v=$ $g(t)-a(t)$, so that $\|v\| \leqq 1+\delta^{\prime}(\varepsilon / 2)$ and $1+\delta^{\prime}(\varepsilon) \geqq\|u\|>\|v\|$ and we want to show that going a distance of $2 \varepsilon$ from $u$ towards $v$, we enter the $\left(1+\delta^{\prime}(\varepsilon / 2)\right)$-ball around 0 . Since this is true if $\|v\|=0$, 
it suffices to show it when $\|v\|=1+\delta^{\prime}(\varepsilon / 2)$.

In the 2-dimensional space spanned by $u$ and $v$ let $z$ be on the $\|v\|$-sphere, on the same side of the line through 0 and $u$ as $v$ is, such that the line $\overline{u z}$ supports the sphere. Extend this line to a hyperplane $H=\psi^{-1} 1$ supporting the $\|v\|$-ball in $E$. Let $\phi=\|v\| \psi, x=u /\|u\|$, $y=z /\|z\|$. Then $\|\phi\|=\phi(y)=1=\|y\|=\|x\|$ and $\dot{\phi}(x)=1 /\|u\| \geqq$ $1 /\left(1+\delta^{\prime}(\varepsilon)\right)>1-\delta^{\prime}(\varepsilon)$, hence $\|x-y\|<\varepsilon$ and $\|u-z\|<\varepsilon+\|u-x\|+$ $\|z-y\| \leqq \varepsilon+\delta^{\prime}(\varepsilon)+\delta^{\prime}(\varepsilon / 2)<2 \varepsilon$. This proves our claim, since the distance from $u$ to the $\|v\|$-ball in the direction of $v$ is less than the maximal of the distances in the directions of $x$ (which is $\leqq \delta^{\prime}(\varepsilon)$ ) and $z$ (which is $<2 \varepsilon$ ).

Inductively, we find $f_{n+1}$ with $\left\|f_{n+1}-f_{n}\right\| \leqq 2 \varepsilon / 2^{n}$ and $r\left(f_{n+1}, A\right) \leqq$ $1+\delta^{\prime}\left(\varepsilon / 2^{n+1}\right)$. The Cauchy sequence $\left(f_{n}\right)$ converges to some $f$ with $\left\|f-f_{0}\right\| \leqq 2 \varepsilon . \quad r(f, A) \leqq \lim r\left(f_{n}, A\right) \leqq 1$, hence $r(f, A)=1$ and $f$ is a Chebyshev center for $A$. (See Remark 6.)

3. Corollary. If $X=C(T, E), E$ a uniformly convex Banach space, then the mapping $A \rightarrow Z(A)$ in $\mathscr{B}(X)$ is uniformly continuous on bounded subsets of $\mathscr{B}(X)$.

Proof. By the proof of Theorem 1, if $r\left(f_{0}, A\right) \leqq\left(1+\delta^{\prime}(\varepsilon)\right) r(A)$, then there is $f \in Z(A)$ with $\left\|f-f_{0}\right\| \leqq 4 \varepsilon r(A)$. Given $R$ and $\varepsilon>0$, let $0<\delta \leqq R \delta^{\prime}(\varepsilon) / 2$. If $r(A) \leqq R, r(B) \leqq R$ and $h(A, B)<\delta$, then for every $x$ we have $|r(x, A)-r(x, B)|<\delta$ (given $u \in A$, find $v \in B$ with $d(u, v)<\delta$ and then $d(x, u)<d(x, v)+\delta$ etc. $)$, hence $|r(A)-r(B)|<$ $\delta$, and for every $z \in Z(A)$ we have $r(z, B)<r(A)+\delta<r(B)+2 \delta \leqq$ $\left(1+\delta^{\prime}(\varepsilon)\right) r(B)$. Therefore we can find $w \in Z(B)$ with $\|w-z\| \leqq 4 \varepsilon R$. Similarly $\sup _{w \in Z(B)} d(w, Z(A)) \leqq 4 \varepsilon R$, i.e., $h(Z(A), Z(B)) \leqq 4 \varepsilon R$.

4. Corollary. If $X=C(T)$ and $Y$ is a closed linear sublattice of $X$, then for every bounded $A \subset X$ there is a relative Chebyshev center in $Y$, and $A \rightarrow Z_{Y}(A)$ is uniformly continuous on bounded subsets of $\mathscr{B}(X)$.

Proof. In the proof of the theorem, if $f_{0}$ and $g$ are chosen in $Y$, then by the lattice property also $f_{1} \in Y$.

The continuity property of the operation $A \rightarrow Z(A)$ in the "most square" space $X=C(T)$, obtained in Corollary 3, is somewhat surprising in view of the next theorem.

5. THeOREM. A Banach space $X$ is uniformly convex iff for every $A \in \mathscr{B}(X) Z(A)$ is a singleton, and $A \rightarrow Z(A)$ is uniformly continuous on bounded subsets of $\mathscr{B}(X)$. 
Proof. Assume first that $X$ is uniformly convex. Since $X$ is reflexive $Z(A) \neq \varnothing$ for every $A \in \mathscr{B}(X)$, while uniform convexity guarantees that $Z(A)$ is a singleton ([1]). It is known (and easily proved) that if $\|x\|,\|y\| \leqq M$ and $\|x-y\| \geqq \varepsilon$, then $\|(x+y) / 2\| \leqq$ $(1-\delta(\varepsilon / M)) M$. Suppose now $z=Z(A), \quad w=Z(B), \quad r(A)<R$ and $h(A, B)<\eta<1$. Then $r(B) \leqq r(z, B)<r(z, A)+\eta=r(A)+\eta$, and $r(A) \leqq r(w, A)<r(B)+\eta<r(A)+2 \eta$. Therefore for $u \in A$ we have $\|u-z\| \leqq r(A),\|u-w\|<r(A)+2 \eta$ and

$$
\left\|u-\frac{z+w}{2}\right\|=\left\|\frac{(u-z)+(u-w))}{2}\right\| \leqq\left(1-\delta\left(\frac{\varepsilon}{R+2}\right)\right)(r(A)+2 \eta) .
$$

But $\|u-(z+w) / 2\| \geqq r(A)$, for some $u \in A$, hence if $\|z-w\| \geqq \varepsilon$ then

$$
\eta \geqq \frac{r(A) \delta\left(\frac{\varepsilon}{R+2}\right)}{2\left(1-\delta\left(\frac{\varepsilon}{R+2}\right)\right)} \geqq \frac{r(A)}{2} \varepsilon\left(\frac{\varepsilon}{R+2}\right) .
$$

Thus if $\eta<r(A) \delta(\varepsilon /(R+2)) / 2$ then $\|z-w\|<\varepsilon$. So that fixing $\eta=$ $\varepsilon \delta(\varepsilon /(R+2)) / 4$ we have either $r(A) \geqq \varepsilon / 2$ and then $\|z-w\|<\varepsilon$, or $r(A)<\varepsilon / 2$ and then taking any $u \in A$ we have $\|z-w\| \leqq\|z-u\|+$ $\|u-w\|<r(A)+r(A)+2 \eta<\varepsilon$.

Conversely, if $E$ is not uniformly convex, there are $x_{n}, y_{n} \in E$ with $\left\|x_{n}\right\|=\left\|y_{n}\right\|=1,\left\|x_{n}-y_{n}\right\|=\varepsilon$ and $\left\|x_{n}+y_{n}\right\| \rightarrow 2$. Let

$$
\begin{gathered}
z_{n}=\frac{x_{n}+y_{n}}{\left\|x_{n}+y_{n}\right\|}, \quad A_{n}=\operatorname{conv}\left\{x_{n}, \frac{x_{n}+y_{n}}{2},-\frac{x_{n}+y_{n}}{2},-y_{n}\right\}, \\
B_{n}=\operatorname{conv}\left\{x_{n}, z_{n},-z_{n},-y_{n}\right\} .
\end{gathered}
$$

Then $h\left(A_{n}, B_{n}\right) \rightarrow 0$, but $\left(x_{n}-y_{n}\right) / 4 \in Z\left(A_{n}\right)$ while $0 \in Z\left(B_{n}\right)$. Thus if $Z\left(A_{n}\right)$ and $Z\left(B_{n}\right)$ are singletons, we have $h\left(Z\left(A_{n}\right), Z\left(B_{n}\right)\right)=$ $\left\|\left(x_{n}-y_{n}\right) / 4\right\|=\varepsilon / 4$.

REMARKS. (1) By the proof it is clear that it is enough to check the uniform continuity of $A \rightarrow Z(A)$ on the 2-dimensional subsets $A$ of the unit ball of $X$.

(2) There are nonuniformly convex spaces in which $Z(A)$ is a singleton for every bounded nonempty $A$. It is known ([1]) that if $X$ is reflexive then $Z(A) \neq \varnothing$ for every $A \in \mathscr{B}(X)$ while the condition that $Z(A)$ is at most a singleton for every $A \in \mathscr{B}(X)$ is equivalent to $X$ being u.c.e.d (uniformly convex in every direction, which means that $\delta_{z}(\varepsilon) \equiv \inf \{1-\|(x+y) / 2\| ;\|x\|=\|y\|=1, x-y=$ $\lambda z,\|x-y\| \geqq \varepsilon\}>0$ for every $z \neq 0$ ). Since every separable space can be equivalently renormed to be u.c.e.d ([6]) while only super- 
reflexive spaces can be renormed to be uniformly convex ([2]), every reflexive but nonsuperreflexive separable $X$ can be renormed so that $Z(A)$ is a singleton for every $A \in \mathscr{B}(X)$, while $A \rightarrow Z(A)$ is not uniformly continuous.

(3) If we wish to drop the condition that $Z(A)$ is a singleton, the same proof yields.

"A normed space $X$ is uniformly convex iff every selection for the set-valued map $Z: A \rightarrow Z(A)$ is uniformly continuous on bounded subsets of the domain of definition of $Z$ in $\mathscr{B}(X)$."

Indeed, $A_{n}$ and $B_{n}$ in the proof above have Chebyshev centers, while continuity of every selection of $Z$ implies that $Z$ is singlevalued. Again, we may restrict ourselves to 2-dimensional sets of the type $A_{n}, B_{n}$.

(4) Say that $X$ is "uniformly convex with respect to $Y$ ", where $Y$ is a closed subspace of $X$, if $\delta_{Y}(\varepsilon) \equiv \inf \{1-\|(x+y) / 2\|$; $\|x\|=\|y\|=1,\|x-y\| \geqq \varepsilon, x-y \in Y\}>0$ for every $\varepsilon>0$. The same proof will yield: The Banach space $X$ is uniformly convex with respect to its subspace $Y$ iff $A \rightarrow Z_{Y}(A)$ is a locally uniformly continuous function from $\mathscr{B}(X)$ (or even the 2-dimensional sets in $\mathscr{B}(X))$ to $Y$.

(5) A related result is the following theorem of P. Smith (cf. [7], p. 188): If $E$ is an " $E$-space" (i.e., a Banach space with a Fréchet differentiable dual or, equivalently, a reflexive strictly convex space in which $\left.x_{n} \stackrel{w}{\rightarrow} x,\left\|x_{n}\right\| \rightarrow\|x\| \Rightarrow x_{n} \rightarrow x\right)$ then $A \rightarrow Z(A)$ is a continuous single-valued map from the space of compact subsets of $E$ (in the Hausdorff metric) into $E$.

(6) Theorem 2 has been obtained, independently, by Ka-Sing Lau, who proved in a similar way the following more general result: For any bounded set-valued map $\phi$ from a topological space $X$ into a uniformly convex Banach space $E$ and for every closed $C(X)$ submodule $M$ in $C(X, E)$ there is a best approximation from $M$ to $\dot{\phi}$.

\section{REFERENCES}

1. A. L. Garkavi, The best possible net and the best possible cross section of a set in a normed space, Izv. Akad. Nauk. SSSR, 26 (1962), 87-106 (Russian).

2. R. C. James, Some self dual properties of normed linear spaces, Annals of Math. Studies, 69 (1972).

3. I. M. Kadets and V. Zamyatin, Chebyshev centers in the space $C[a, b]$, Teoria Funk., Funkcion. Anal. Pril., 7 (1968), 20-26 (Russian).

4. A. F. Ruston, $A$ note on convexity of Banach spaces, Proc. Cambridge Philos. Soc., 45 (1949), 157-159.

5. J. D. Ward, Chebyshev centers in spaces of continuous functions, Pacific J. Math., 52 (1974), 283-287.

6. V. Zizler, Rotundity and smoothness properties of Banach spaces, Rozprawy Matem., 87 (1971), 3-33. 
7. R. B. Holmes, A course in optimization and best approximation, Springer Lecture Notes No. 257 (1972).

Received July 12, 1977.

Tel Aviv University

Tel Aviv, IsRael 


\section{PACIFIC JOURNAL OF MATHEMATICS}

\section{EDITORS}

RICHARD ARENS (Managing Editor)

University of California

Los Angeles, California 90024

C. W. Curtis

University of Oregon

Eugene, OR 97403

C. C. MOORE

University of California

Berkeley, CA 94720

\section{J. DUGUNDJI}

Department of Mathematics University of Southern California Los Angeles, California 90007

R. Finn AND J. Milgram Stanford University Stanford, California 94305

\section{ASSOCIATE EDITORS}

E. F. BeCK ENBACH

B. H. NeUMaNN

F. WOLF

K. Yoshida

\section{SUPPORTING INSTITUTIONS}

UNIVERSITY OF BRITISH COLUMBIA CALIFORNIA INSTITUTE OF TECHNOLOGY UNIVERSITY OF CALIFORNIA MONTANA STATE UNIVERSITY UNIVERSITY OF NEVADA, RENO NEW MEXICO STATE UNIVERSITY OREGON STATE UNIVERSITY UNIVERSITY OF OREGON
UNIVERSITY OF SOUTHERN CALIFORNIA STANFORD UNIVERSITY UNIVERSITY OF HAWAII UNIVERSITY OF TOKYO UNIVERSITY OF UTAH WASHINGTON STATE UNIVERSITY UNIVERSITY OF WASHINGTON 


\section{Pacific Journal of Mathematics \\ Vol. 77, No. $1 \quad$ January, 1978}

Dan Amir, Chebyshev centers and uniform convexity ............... 1

Lawrence Wasson Baggett, Representations of the Mautner group. I ..... 7

George Benke, Trigonometric approximation theory in compact totally

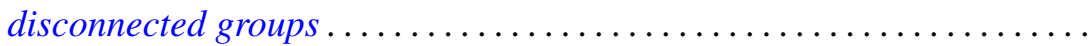

M. Bianchini, O. W. Paques and M. C. Zaine, On the strong compact-ported topology for spaces of holomorphic mappings ..................

Marilyn Breen, Sets with $(d-2)$-dimensional kernels

J. L. Brenner and Allen Kenneth Charnow, Free semigroups of $2 \times 2$

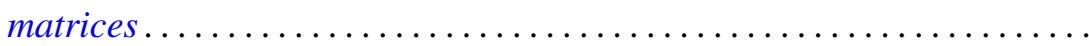

David Bressoud, A new family of partition identities .................

David Fleming Dawson, Summability of matrix transforms of stretchings

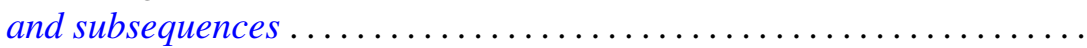

Harold George Diamond and Paul Erdôs, A measure of the nonmonotonicity

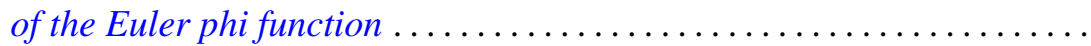

Gary Doyle Faulkner and Ronald Wesley Shonkwiler, Kernel dilation in reproducing kernel Hilbert space and its application to moment

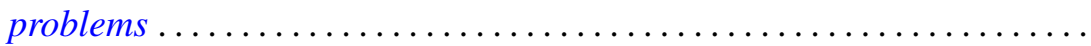

Jan Maksymilian Gronski, Classification of closed sets of attainability in the

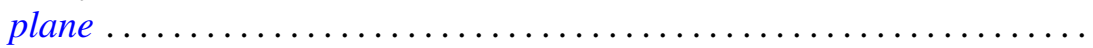

H. B. Hamilton and T. E. Nordahl, Semigroups whose lattice of congruences

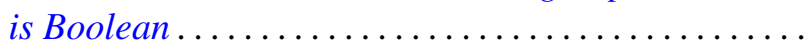

Harvey Bayard Keynes and D. Newton, Minimal $(G, \tau)$-extensions ...

Anthony To-Ming Lau, The Fourier-Stieltjes algebra of a topological

semigroup with involution.

B. C. Oltikar and Luis Ribes, On prosupersolvable groups ...

Brian Lee Peterson, Extensions of pro-affine algebraic groups ...

Thomas M. Phillips, Primitive extensions of Aronszajn spaces ...

Mehdi Radjabalipour, Equivalence of decomposable and 2-decomposable operators. .

M. Satyanarayana, Naturally totally ordered semigroups .

Fred Rex Sinal, A homeomorphism classification of wildly imbedded two-spheres in $S^{3}$

Hugh C. Williams, Some properties of a special set of recurring

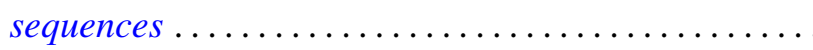

